\title{
CONHECIMENTO DE ARQUITETURA DE PRODUTO COMO ELEMENTO CHAVE PARA A MANUTENÇÃO DA CAPACIDADE INOVADORA DE UMA EMPRESA - O CASO DA INDÚSTRIA AUTOMOTIVA
}

\section{PRODUCT ARCHITECTURAL KNOWLEDGE AS KEY ELEMENT TO INNOVATIVE CAPACITY MAINTENANCE - THE CASE OF AUTOMOTIVE INDUSTRY}

\author{
Adriana Marotti de Mello ${ }^{1}$; Roberto Marx ${ }^{2}$ \\ ${ }^{1}$ Escola Politécnica da Universidade de São Paulo - Brasil adriana.mello@poli.usp.br \\ ${ }^{2}$ Escola Politécnica da Universidade de São Paulo - Brasil robemarx@usp.br
}

\begin{abstract}
Resumo
O Conhecimento de arquitetura de produto pode ser definido como o conhecimento requerido para unir diferentes módulos e componentes em um sistema coerente, com funcionalidade integrada. Este conhecimento seria fundamental para transformar em especificações funcionais de produtos os desejos e necessidades do mercado consumidor e assim, manter a capacidade de uma empresa inovar em determinado mercado, a partir de um dado paradigma tecnológico. Através de um estudo de caso sobre o desenvolvimento de um dos veículos populares mais vendidos no Brasil, este trabalho procura discutir como este conhecimento influencia a manutenção da capacidade de inovar de uma montadora de veículos. A conclusão é de que o conhecimento de arquitetura ainda é uma competência exclusiva das montadoras. É através desta competência que a montadora continua capaz de detectar as necessidades e desejos do mercado e traduzi-las especificações de um produto que as atenda. E os fornecedores, dentro desse mesmo paradigma tecnológico, não possuiriam essa competência.
\end{abstract}

Palavras-chave: Desenvolvimento de Produtos, Indústria Automotiva, Arquitetura de Produto.

\section{Introdução}

Na busca pela vantagem competitiva sustentável, as empresas têm concentrado seus esforços no sentido de buscar uma organização mais eficiente, seja em custos, seja em agilidade no atendimento das demandas do mercado.

Nesse cenário, o Desenvolvimento de Novos Produtos (doravante DP) tem fundamental importância estratégica na definição da competitividade dentro da indústria. Há a necessidade de encurtar prazos de desenvolvimento, diversificar o portfolio de produtos e reduzir custos. 
Diferentes empresas em diferentes setores procuraram formas alternativas de organizar o gerenciamento da produção e o desenvolvimento de produtos, mas uma das formas de organizá-los que parece emergir com muita força como paradigma nos últimos anos, é a externalização de atividades para fornecedores (outsourcing) (Clark e Fujimoto, 1991). Uma das decisões mais importantes para a empresa diz respeito a quais atividades externalizar e quais fazer internamente (decisão make-buy) e como coordenar as atividades inter-firma no desenvolvimento de produtos.

É bastante discutido no meio acadêmico e gerencial o caso da IBM e de como ela perdeu seu poder dentro da indústria de computadores após o lançamento do microcomputador pessoal (PC). A IBM era uma empresa altamente verticalizada, desenvolvendo internamente todos seus componentes. No início da década de 80 , para acelerar o desenvolvimento do PC, a IBM adotou um projeto de estrutura modular, definiu regras e parâmetros para integrar os diferentes módulos e delegou a fornecedores o desenvolvimento de módulos como microprocessadores (para a Intel) e o sistema operacional (para a Microsoft).

Essa estratégia desverticalizada tornou-se dominante no setor, e outras empresas passaram a produzir PC's da mesma maneira. Os clientes passaram a comprar não mais um PC IBM, mas um PC compatível com padrão Intel / Windows. A conseqüência passou a ser que o poder na cadeia de suprimento, ou seja, o elemento que passou a determinar a evolução no setor, foi não mais o fabricante do PC completo, mas de um de seus componentes (a Intel, com o microprocessador). Essa é a chamada "síndrome Intel-inside”. (Fine, 1998).

$\mathrm{Na}$ indústria automotiva verifica-se a tendência de externalizar o Desenvolvimento de Produtos, o que poderia levar à hipótese de que as montadoras estariam, assim como a IBM, perdendo sua capacidade inovadora de desenvolver novos produtos que atendam às demandas do mercado onde elas atuam, tornando-se cada vez mais dependentes de seus fornecedores para fazêlo. Contudo, até o presente momento não se verifica tendência similar à da indústria de computadores, ou seja, são as montadoras de automóveis que ainda mantêm a capacidade de inovar em produtos, mesmo com a externalização de atividades de desenvolvimento para fornecedores. (Mello, Vasconcellos e Marx, 2005).

A diferença entre o ocorrido na indústria de computadores e na automotiva estaria no domínio do conhecimento de arquitetura, por parte da empresa que externaliza o DP, ou seja, do conhecimento de como integrar diferentes componentes em um sistema coerente (Henderson e Clark, 1990). Esse conhecimento dependeria da empresa possuir conhecimento específico sobre a tecnologia dos componentes de seus produtos e, principalmente, do domínio das interfaces entre esses componentes (Takeishi, 2002). A empresa que domina o conhecimento de arquitetura do produto manteria sua capacidade de inovar dentro dessa mesma arquitetura. 
O conhecimento de arquitetura seria de importância fundamental para a capacidade de inovar em automóveis, uma vez que a percepção dos clientes do que é realmente inovador é ligado mais à funcionalidade do conjunto do que da funcionalidade de módulos e componentes em separado (Fixson e Sako, 2001).

Este artigo tem por objetivo discutir a questão de como o domínio do conhecimento de arquitetura interfere na manutenção da capacidade inovadora das empresas, através de pesquisa sobre o processo de desenvolvimento do VW Fox, especialmente no que se refere a estratégia de envolvimento de fornecedores. Será também pesquisado o processo de desenvolvimento de dois módulos: o cockpit e os bancos, com o objetivo de aprofundar a pesquisa e incluir a visão dos fornecedores sobre o assunto.

O presente artigo está organizado da seguinte maneira: na Seção 2, apresenta-se a revisão bibligráfica realizada sobre o conceito de conhecimento de arquitetura e qual a influência deste conceito na manutenção da capacidade inovadora de uma empresa, mesmo com a externalização de atividades de desenvolvimento; a Seção 3 traz a metodologia de pesquisa; na Seção 4, são apresentados os resultados da pesquisa empírica realizada na Seção 5, discutem-se os resultados e traz-se a conclusão do trabalho. A Seção 6 é dedicada a apresentar as limitações da pesquisa realizada e sugerir novas pesquisas.

\section{Domínio do Conhecimento de Arquitetura como forma de manter a Capacidade Inovadora de uma empresa}

Para melhor compreensão desse fator e de como ele afeta a capacidade inovadora de uma montadora, é necessário primeiro definir o que é a Arquitetura de Produto e o que é o Conhecimento de Arquitetura, o que será feito a seguir:

Um produto pode ser imaginado tanto do ponto de vista funcional, como em termos físicos. Os elementos funcionais são as operações individuais e transformações que contribuem para a performance do produto com um todo. Os elementos físicos são as peças, os componentes e subconjuntos que implementam as funções requeridas. (Ulrich e Eppinger, 95).

Os elementos físicos normalmente são agrupados em blocos (chunks), que implementam as funções no produto. A arquitetura do produto é a forma em que a funcionalidade do produto é agrupada pelos blocos físicos.

A arquitetura de um produto pode ser modular e/ou integral:

Um produto possui arquitetura integral quando os elementos funcionais são implementados usando mais de um bloco, ou um bloco implementa várias funções. As interações entre os blocos não são bem definidas. Ele é concebido visando alta performance e a 
fronteira entre os blocos é de difícil identificação, senão inexistente. A mudança de um componente pode exigir um novo projeto de produto.

Um produto possui arquitetura modular quando seus blocos físicos implementam um ou poucos elementos funcionais e suas interações são bem definidas e geralmente fundamentais para as funções primordiais do produto. A arquitetura modular permite a mudança de projeto de um módulo de forma independente, ou seja, sem a necessidade de mudança em outros módulos.

Um produto raramente pode ser classificado como estritamente modular ou integral. Ele pode ser classificado relativamente a outros produtos de acordo com seu grau de modularidade (Ulrich e Eppinger, 1995).

O tipo de arquitetura de um produto é decidido ao longo de seu desenvolvimento e esta definição vai afetar sua performance e variedade de versões, influenciar sua forma de modificação posterior ao desenvolvimento, reduzir ou aumentar o tempo de desenvolvimento e, principalmente, afetar a forma que o processo de desenvolvimento de produto pode ser gerenciado.

Um produto de arquitetura modular permite que diferentes grupos trabalhem independentemente no desenvolvimento destes, uma vez que trabalham com um número relativamente limitado e conhecido de interações com outros módulos. Já um produto de arquitetura integral, requer um envolvimento e coordenação muito grande entre os grupos. Por isso, para um produto de arquitetura modular é mais simples a delegação da responsabilidade pelo desenvolvimento e produção de módulos para diferentes empresas, como no caso de um microcomputador, por exemplo, onde o micro-processador, o hard-disk e o monitor, entre outros, são desenvolvidos e produzidos por diferentes empresas, e montados por uma terceira empresa (Baldwin e Clark, 1997).

Um automóvel tanto pode ser encarado como um produto de arquitetura integral, se considerarmos que algumas funções do automóvel estão dispersas em diferentes blocos - como a velocidade, por exemplo: não só a performance do motor, mas da transmissão, os pneus, a aerodinâmica, a carroceria e seu peso afetam essa função - quanto produto de arquitetura modular ele pode ser entendido como um conjunto de módulos, componentes e sub-sistemas com funções específicas definidas (painel de instrumentos, motor, câmbio, etc.).

A diferença entre um produto visto como um sistema e um produto visto como um conjunto de componentes ilustra a idéia dos dois tipos de conhecimento requeridos no DP: o conhecimento de componentes e o conhecimento de arquitetura. O primeiro é o conhecimento dos conceitos básicos de design do produto e de como estes conceitos são implementados em cada componente em particular.

Pode-se definir conhecimento de arquitetura como: 
"O conhecimento de como os diferentes componentes são integrados formando um conjunto coerente, um sistema com funcionalidade integrada, e não simplesmente um conjunto de componentes..." (Henderson e Clark, 1990).

O conhecimento de arquitetura surge, portanto, depois que uma organização desenvolveu familiaridade suficiente com a interação dos diferentes componentes que permita que ela possa fragmentar um produto em elementos menores sem que com isso, perca informação crítica da funcionalidade do produto como um todo. Esse conceito de conhecimento de arquitetura é fundamental para se entender o papel da empresa que integra diferentes módulos em um produto com funcionalidade única e foi criado por Henderson e Clark (1990).

Nos estágios iniciais da evolução tecnológica de um setor, há grande experimentação, com diferentes soluções tecnológicas para um mesmo produto. Uma vez que um conceito de produto é estabelecido como dominante, o conjunto inicial de componentes é refinado e elaborado e o progresso tecnológico caminha na direção do desenvolvimento do conceito de cada componente individualmente dentro de uma arquitetura estável, em torno da qual é organizado o setor. Por exemplo, no início do desenvolvimento dos automóveis, foram desenvolvidas diferentes motorizações (usando gasolina, eletricidade ou vapor como combustível) e diferentes soluções de tração e carroceria (metal ou madeira). Após a consolidação do conceito dominante de veículo carroceria metálica, com motor de combustão interna e tração traseira - o desenvolvimento do setor foi no sentido de refinar a concepção tecnológica dos componentes dessa arquitetura (Henderson e Clark, 1990).

$\mathrm{O}$ conhecimento de arquitetura está diretamente ligado à capacidade de uma empresa de identificar diferentes necessidades funcionais e traduzi-las em especificações técnicas, alocando essas funções nos diferentes módulos que compõe o produto e especificando as interfaces entre esses módulos (Fixson e Sako, 2001).

Em um produto com arquitetura modular, mas com funções dispersas por vários módulos ou sistemas, é fundamental a presença de um integrador desses módulos, um coordenador que domine o conhecimento de arquitetura, que define e ajuste as regras do projeto que determinam interconexões entre as interfaces dos módulos. Nesse ajuste, que muitas vezes ocorre de forma iterativa, o conhecimento dos módulos e seus componentes é necessário. Um exemplo disto é a especificação máxima de ruído de vibração em um automóvel (NVH) em diferentes velocidades. Para conseguir atingir essa especificação, é necessário conhecimento das inter-relações entre a carroceria, o chassis e o conjunto motriz do veículo (Sako, 2002).

O domínio do conhecimento de arquitetura pode ser definido como a "raison d'être" das montadoras de automóveis e a principal fonte de sua vantagem competitiva (Takeishi, 2002). É este domínio que dá às montadoras a capacidade de identificar as necessidades e desejos dos clientes, 
transformando-a em especificações funcionais de produtos. Perdendo o controle sobre esse conhecimento, poder-se-ia afirmar que uma empresa perderia sua capacidade de inovar. É o que ocorreu com a IBM, no desenvolvimento do PC: ao dotar o produto de uma arquitetura modular, mas com interfaces padronizadas, e com o desenvolvimento de componentes externalizados para fornecedores, a IBM perdeu o controle sobre o conhecimento de arquitetura: este conhecimento ficou disperso entre os diferentes componentes e diferentes fornecedores.

Esse mesmo fenômeno ocorreu com a indústria de bicicletas, de acordo com o trabalho de Ulrich e Ellison (2004): a capacidade de inovar em produtos, hoje pertence às empresas que fabricam componentes como o câmbio e sistemas de freio (a Shimano, a "Intel das bicicletas"). A função de integrar os diferentes componentes em um único produto não é mais crítica para a inovação do produto, uma vez que a arquitetura de produto é modular, e seus componentes e interfaces são padronizados.

Já no caso dos automóveis, não há uma correlação única entre funcionalidade e sua localização em um determinado módulo ou componente. Funções e características próprias de cada modelo como velocidade, conforto acústico, por exemplo, estão dispersas entre diversos módulos e componentes, o que faz com que o conhecimento de arquitetura seja fundamental na capacidade de inovar em produtos nesta indústria. Portanto, quem controla o conhecimento de arquitetura, possuiria a capacidade de inovar.

\section{Metodologia da pesquisa}

A externalização do DP no Brasil, como estratégia para obter vantagem competitiva sustentável, vem se intensificando desde os anos 1990 e seus impactos sobre a base de conhecimento das empresas ainda é recente e pouco conhecido. Além disso, é um processo complexo e lento, que envolve criação, dispersão e coordenação de conhecimento.

Por isso, pode-se afirmar que o estudo dos impactos da dispersão do conhecimento e de como as empresas que externalizam o DP estão lidando com isto ainda está na fase de construção de teoria. O uso de estudo de caso como método de pesquisa é particularmente útil quando ainda não há certeza na definição dos constructos e variáveis que constroem a teoria que explicaria um fenômeno(Voss et al,2002).

A pesquisa realizada neste trabalho será, portanto, de natureza qualitativa e será conduzida pelo método de estudo de caso. No entanto, a pesquisa qualitativa apresenta algumas desvantagens. A primeira delas é uma maior dificuldade na determinação da validade e confiabilidade dos resultados (Miles e Huberman, 1994). Uma segunda desvantagem deste tipo de pesquisa é a verificação de que os projetos qualitativos podem se tornar muito complexos, com um nível de 
detalhe muito profundo, dificultando a identificação das relações mais importantes para a construção de uma teoria. Por último, a pesquisa qualitativa através de estudo de casos pode levar a conclusões não generalizáveis, por se estar analisando apenas uma parte do fenômeno. (Eisenhardt, 1989).

Apesar destas desvantagens, a pesquisa qualitativa conduzida através de estudo de caso é a forma mais indicada, segundo Eisenhardt (1989), para pesquisas em seus estágios iniciais em que há pouco conhecimento sobre um fenômeno. Os métodos de pesquisa qualitativa conduzidos através de estudo de caso são indicados para responder perguntas do tipo "como" e "por que" de um dado fenômeno (Yin, 2002).

O objeto de análise foi o processo de desenvolvimento de um veículo popular, especialmente no que se refere a estratégia de modularização e envolvimento de fornecedores. A empresa objeto do estudo foi a Volkswagen. O motivo da escolha está relacionado ao fato da empresa, em princípio, parecer apresentar uma área de desenvolvimento de produto sintonizada às necessidades dos clientes, o que posteriormente pôde ser observado pela boa aceitação de mercado do produto desenvolvido e pelo fato da empresa desenvolver localmente veículos.

Os módulos escolhidos para realização do estudo - cockpit e bancos - têm alto valor agregado, são tecnologicamente complexos (envolvem diferentes tipos de tecnologia na sua fabricação - como injeção de termoplásticos, soldagem, eletrônica, materiais têxteis, por exemplo), têm alto impacto na percepção da qualidade do produto pelos clientes e contam com envolvimento de fornecedores no seu desenvolvimento em variados graus no projeto objeto de estudo. Estas características dos módulos escolhidos facilitam a análise da pergunta a que este artigo procura responder.

Inicialmente, foi realizado um levantamento bibliográfico nas áreas de: Inovação, Desenvolvimento de Produtos, Arquitetura de Produto e Conhecimento de Arquitetura.

Seguindo procedimentos propostos por Yin (2002) na coleta de dados, para o estudo do caso foram utilizadas três fontes de coleta de dados, a saber, documentação, entrevistas e observação direta.

Pode-se afirmar que as fontes de informação mais importantes utilizadas neste estudo foram as entrevistas. Utilizando-se questionários pré-estruturados, foram entrevistados individualmente executivos da montadora e das empresas fornecedoras do módulo banco e da cobertura do painel de instrumentos. Estes executivos pertencem às áreas de Desenvolvimento de Produto, Comercial e Qualidade. As entrevistas foram gravadas (quando permitidas pelo entrevistado) e depois transcritas na sua íntegra para melhor compreensão do material obtido.

A observação é outra forma de coletar evidências para o estudo de caso segundo Yin (2002). De uma maneira informal, foram realizadas observações diretas durante as visitas de campo para 
coletar evidências através de entrevistas. O levantamento observatório foi útil para fornecer informações adicionais sobre os tópicos do estudo.

\section{Estudo de Caso - O desenvolvimento do VW Fox}

Lançado no mercado nacional em 2003, o VW Fox foi inteiramente desenvolvido no Brasil, sobre a plataforma PQ 24 (do Polo), com a missão de ser um veículo de baixo custo, como forma da VW enfrentar a crescente concorrência no segmento. O desafio foi desenvolver o mais rápido e barato possível o veículo para conseguir a aprovação da matriz. Hoje o Fox é fabricado nas fábricas de S.José dos Pinhais (juntamente com o Golf) e na fábrica da Anchieta (na nova linha do Polo). É também exportado para a América Latina e Europa.

O conceito de produto e processo para o Fox é modularizado, mas comparando-se seu nível de modularização com outros veículos montados pela mesma empresa, como o Polo e o Gol, verifica-se que seu nível de modularização está mais próximo do Gol do que o do Polo. A razão para este fato, é que o foco do projeto era a minimização dos custos de produto e determinados módulos como o front-end, a porta e os chicotes foram considerados pela empresa mais baratos para o Fox se fossem entregues separadamente, como no caso do Gol.

TABELA 1 - Divisão em Módulos do Fox, Gol e Polo

\begin{tabular}{|l|c|c|c|}
\hline \multicolumn{1}{|c|}{ Módulo } & Fox & Gol & Polo \\
\hline Front-end & & & $\mathrm{X}$ \\
\hline Cockpit & $\mathrm{X}$ & & $\mathrm{X}$ \\
\hline Bancos (diant/tras) & $\mathrm{X}$ & $\mathrm{X}$ & $\mathrm{X}$ \\
\hline Porta & & & $\mathrm{X}$ \\
\hline Chicotes & & & $\mathrm{X}$ \\
\hline Suspensão diant & $\mathrm{X}$ & $\mathrm{X}$ & $\mathrm{X}$ \\
\hline Suspensão traseira & $\mathrm{X}$ & $\mathrm{X}$ & $\mathrm{X}$ \\
\hline Tanque combustível & $\mathrm{X}$ & & $\mathrm{X}$ \\
\hline
\end{tabular}

Fonte: Elaborada pela autora, a partir de dados da pesquisa

\section{O módulo Cockpit - Projeto VW Fox}

O Cockpit é entregue como módulo diretamente na linha de montagem para o Fox. Na fábrica de São José dos Pinhais (onde são montados o Golf e o Fox mercado nacional), é montado e entregue por um fornecedor localizado dentro da planta. Na fábrica Anchieta (para o Polo e Fox Exportação), é montado pela própria VW. Já para o Gol, as peças são entregues separadamente e montadas já no veículo, sem conceito de módulo, devido ao conceito de projeto de produto e 
processo. Apresenta-se os componentes do módulo cockpit para o Fox na tabela abaixo:

TABELA 2 - Principais constituintes do módulo cockpit e responsabilidade no desenvolvimento

\begin{tabular}{|l|l|}
\hline $\begin{array}{l}\text { Principais constituintes do } \\
\text { módulo cockpit }\end{array}$ & $\begin{array}{l}\text { Envolvimento de fornecedores } \\
\text { no desenvolvimento }\end{array}$ \\
\hline Suporte painel (Träger) & Co-design \\
\hline Cobertura painel & $\begin{array}{l}\text { Desenvolvimento do processo } \\
\text { de produção }\end{array}$ \\
\hline Caixa de ar & Black-box \\
\hline Módulo Air Bag & $\begin{array}{l}\text { Desenvolvimento do processo } \\
\text { de produção }\end{array}$ \\
\hline Volante & $\begin{array}{l}\text { Desenvolvimento } \\
\text { ferramental }\end{array}$ \\
\hline Instrumento Combinado & Black-box \\
\hline Chicotes & Co-design \\
\hline Pedaleira & Co-design \\
\hline Coluna de direção & Co-design \\
\hline Diversas Fixações e Coberturas & $\begin{array}{l}\text { Desenvolvimento de } \\
\text { ferramental ou peças standard } \\
\text { (fixações) }\end{array}$ \\
\hline
\end{tabular}

Fonte: Elaborada pela autora, a partir de dados da pesquisa

Dentre todas as peças, a mais crítica do ponto de vista de qualidade do acabamento, de custo e de montagem para o módulo como um todo, é a cobertura do painel. Ela deve absorver todas as variações de dimensão da carroceria e do suporte do painel, é uma peça de grandes dimensões, de complexa tecnologia de produção (envolve injeção de plástico, solda, montagem de componentes), com alto nível de exigência de qualidade visual (é a principal peça que o comprador sente e vê ao dirigir) e possui interfaces com peças de funcionalidade complexa (como o ar condicionado e o acionamento de todos comandos do motorista).

No desenvolvimento do módulo como um todo - detalhamento de quais peças o comporiam, quais especificações de interface (fixações na carroceria, p.ex) não houve nenhum envolvimento de fornecedores. O fornecedor do módulo para a planta de Curitiba tem contrato apenas de fornecimento de mão de obra e serviços logísticos e desenvolve apenas seu processo de produção.

Cada componente individual tem sua própria estratégia de envolvimento de fornecedores, dependendo de cada projeto (vide tabela 2). A caixa de ar e o instrumento combinado são blackboxes, ou seja, foram desenvolvidos totalmente por seus fornecedores a partir de um caderno de encargos, que contêm as especificações de desempenho requeridas pela montadora. Outras peças, como o suporte do painel, foram desenvolvidas em co-design, ou seja, em parceria da montadora com o fornecedor, mas com a responsabilidade final sobre o projeto da montadora. O envolvimento dos fornecedores também pode ser através do desenvolvimento de ferramental (como no caso das coberturas plásticas), onde a montadora é responsável pelo conceito do produto, e o fornecedor pelo 
desenvolvimento do ferramental e demais meios de produção, ou como o fornecedor é envolvido apenas na fase desenvolvimento do processo de produção, sendo a montadora responsável pelo conceito do produto e desenvlvimento de ferramental.

No caso do Fox, praticamente não houve envolvimento do fornecedor no desenvolvimento da cobertura do painel de instrumentos. Até o ferramental foi desenvolvido pela própria VW. A justificativa foi em função de custo e da definição tardia do fornecedor - como houve uma intensa discussão comercial, a escolha foi retardada. O fornecedor apenas desenvolveu o processo de fabricação da peça. Muitos problemas de especificação da cobertura do painel só puderam ser verificados nesta fase, o que gerou necessidade de modificação de ferramental e consequentemente, aumento no custo de desenvolvimento e atrasos.

\section{O módulo Banco - Projeto VW Fox}

O conjunto de bancos é tratado como o Fox como um módulo: o fornecedor é pago pela mão de obra empregada na montagem dos bancos, não havendo responsabilidade pelo desenvolvimento ou administração dos componentes. Ele é entregue sequenciado diretamente na linha de montagem. Para todas as unidades da VW, os bancos são fornecidos por terceiros. O módulo banco é composto pelos seguintes componentes:

TABELA 3 - Principais constituintes do módulo banco e responsabilidade no desenvolvimento

\begin{tabular}{|l|l|}
\hline $\begin{array}{l}\text { Principais constituintes do } \\
\text { módulo banco }\end{array}$ & $\begin{array}{l}\text { Envolvimento de fornecedores } \\
\text { no desenvolvimento (para o } \\
\text { Fox) }\end{array}$ \\
\hline Estrutura Metálica & Black box \\
\hline Espuma & $\begin{array}{l}\text { Desenvolvimento do processo } \\
\text { de produção }\end{array}$ \\
\hline Capa & $\begin{array}{l}\text { Desenvolvimento do processo } \\
\text { de produção }\end{array}$ \\
\hline Coberturas plásticas & $\begin{array}{l}\text { Desenvolvimento do } \\
\text { ferramental }\end{array}$ \\
\hline Apoio de Cabeça & $\begin{array}{l}\text { Desenvolvimento de } \\
\text { ferramental }\end{array}$ \\
\hline Fixações & Peças padronizadas \\
\hline
\end{tabular}

Fonte: Elaborada pela autora, a partir de dados de pesquisa

No desenvolvimento do Fox, as especificações de interface entre os componentes que compõem o módulo foram todas determinadas pela montadora. O fornecedor do módulo não se envolveu no desenvolvimento do conjunto, nem de seus componentes nas fases iniciais do projeto; seu envolvimento com o processo foi apenas no desenvolvimento do processo de produção. Mas no desenvolvimento do banco, algumas especificações de componentes individuais só podem ser testadas após a montagem completa do módulo. Por este motivo, algumas modificações tiveram de ser realizadas no projeto dos componentes, acarretando sumento de custos para o produto. 
No caso dos componentes, o grau de envolvimento dos fornecedores é variado: há peças black-boxes, onde o projeto é de responsabilidade do fornecedor, como o caso das estruturas dos bancos dianteiros para o veículo 2 portas e dos bancos traseiros corrediços, peças onde o fornecedor desenvolveu o ferramental e onde somente desenvolveu o processo de produção.

A partir da análise dos dados acima, sobre o envolvimento de fornecedores nos dois módulos analisados, pôde-se proceder sua análise. Os dados foram analisados individualmente e comparados entre si. Algumas observações realizadas pelos fornecedores dos módulos sobre diferenças no relacionamento entre a VW e outras montadoras também foram consideradas.

\section{Análise dos Resultados e Conclusões}

$\mathrm{Na}$ descrição do desenvolvimento dos módulos banco e cockpit para o VW Fox, ficou claro que o controle das interfaces entre os diferentes componentes de cada módulo, que dão a funcionalidade integrada de cada um, ainda pertence à montadora.

Para os bancos, mesmo com componentes sendo desenvolvidos por fornecedores, é a VW quem determina o aspecto visual (cores, padronagens de tecidos), especificações funcionais, como torques e especificações de materiais (dureza de espuma, resistência a tração dos tecidos, resistência a radiação UV). O resultado é que características que determinam a percepção de qualidade do cliente tais como conforto e aspecto visual estão sob controle da montadora. Além disso, todos os testes funcionais para homologação do produto, como testes de durabilidade, segurança, rodagem em veículo, são feitos pela montadora. Mesmo quando ela não possui todos os recursos internamente para realizar os testes (como testes de airbag em banco), utiliza-se os recursos do fornecedor, mas sob supervisão e responsabilidade da montadora.

Também para as montadoras que externalizaram o desenvolvimento do banco completo, não há tanta liberdade para o fornecedor criar um produto inteiramente novo: as especificações básicas visuais e funcionais são determinadas pelas montadoras, que podem aceitar ou não inovações e sugestões dos fornecedores.

Da mesma maneira, para o módulo cockpit, o controle das interfaces entre seus diferentes componentes pertence à montadora. Este módulo possui elevada complexidade funcional, integrando diferentes funções como painel de instrumentos, acionamento de diversos comandos do veículo e caixa de ar. Além disso, ele é fundamental para a percepção da qualidade dos clientes.

As palavras de um executivo do fornecedor de painel e módulo cockpit entrevistado ilustram bem o exemplo acima:

“...Cada montadora tem seu próprio conjunto de especificações sobre as quais elas não abrem mão, que são como dogmas, paradigmas de desenvolvimento muito fortes: o ponto $\mathrm{H}$, a 
altura do carro, o posicionamento do motorista, a distância entre o motorista e o painel, por exemplo - estas especificações são aquelas que o cliente identifica como característica própria de cada montadora e nós, fornecedores, desenvolvemos os componentes para atingir estas especificações..."

A análise dos resultados obtidos nas entrevistas, análises de documentos e dados demonstra que o controle das interfaces e o conhecimento de como unir os diferentes módulos e componentes em um produto com funcionalidade integrada fique com a montadora. E o domínio desse conhecimento faz com que a capacidade de inovar no mercado brasileiro de automóveis ainda pertence à montadora, mesmo com a tendência de externalizar atividades de DP para os fornecedores. Ficou claro, também, que as montadoras não mais possuem, ou desejam possuir, capacidade para desenvolver sozinhas um veículo completo, com toda sua complexidade tecnológica. As montadoras encontram-se dependentes em conhecimento, conforme o modelo de Fine e Whitney (1996), especialmente em tecnologias como a eletrônica. O fato de as montadoras estarem dependentes em conhecimento de seus fornecedores não implica diretamente em elas estarem perdendo a capacidade de inovar. Através da pesquisa bibliográfica e dos dados levantados, pode-se concluir que a capacidade de gerenciar a inovação ao externalizar atividades de DP é muito influenciada pela manutenção do controle do conhecimento de arquitetura.

Contudo, é importante discutir o conceito de inovação em arquitetura, proposto por Henderson e Clark (1990). A inovação em arquitetura ocorre quando há uma reconfiguração de um sistema estabelecido de união dos componentes. Um exemplo seria o desenvolvimento de um ventilador portátil a partir de um ventilador de teto. Os componentes básicos do produto (pás, motor, sistemas de controle) permanecem o mesmo, mas a forma de configurá-los em um produto com diferentes requisitos de projeto (tamanho, localização do motor) faz com que as interações entre os componentes sejam alteradas, fazendo com que o conhecimento de arquitetura para o ventilador de teto seja inútil para desenvolver um ventilador portátil. A partir daí, pode-se questionar o que ocorreria com o conhecimento de arquitetura acumulado pelas montadoras se uma inovação, como o motor movido a hidrogênio, por exemplo, alterasse a arquitetura atual do veículo.

Por isso, pode-se concluir que o domínio do conhecimento de arquitetura garante a manutenção da capacidade de inovar somente dentro de um dado paradigma tecnológico - uma inovação em arquitetura pode alterar drasticamente o conhecimento de arquitetura necessário para desenvolver um novo produto e, neste caso, a montadora correria riscos consideráveis de não mais manter esta capacidade de inovar. 


\section{Limitações do Estudo e Futuras Pesquisas}

As limitações deste estudo derivam-se do próprio método utilizado em sua elaboração: pesquisa qualitativa conduzida através de estudo de caso. Em função da limitação de tempo, apenas uma montadora e dois fornecedores foram pesquisados; por esta razão, as conclusões deste trabalho não podem ser generalizáveis, mas contribuem para a discussão do tema dos impactos da externalização do desenvolvimento de produtos na capacidade inovadora das empresas. Uma pesquisa que envolvesse outras montadoras e um número maior de módulos no Brasil seria interessante de ser feita no futuro. Da mesma maneira, pode-se especular se o resultado obtido nesta pesquisa reproduzir-se-ia em mercados automotivos com características diferentes do brasileiro, como o europeu ou americano, onde o conteúdo tecnológico embutido nos veículos é bem maior.

Para uma compreensão mais ampla deste tema, sugere-se no futuro uma pesquisa mais ampla sobre as conseqüências da externalização do DP não só em montadoras de veículos, mas também em outros setores industriais, como o eletrônico, por exemplo.

Além disso, seria interessante verificar como outros fatores tais como a estratégia de produto da empresa, sua trajetória e as características do setor e do mercado onde a empresa atua, por exemplo, podem afetar a manutenção da capacidade inovadora. O desenvolvimento de uma teoria sobre esse assunto seria muito importante para a área de Gestão de Tecnologia e Operações e também para praticantes do setor industrial.

\section{Abstract}

The Architectural Knowledge of a product can be defined as the required knowledge to integrate different modules and components into a coherent system, with integrated functionality. This kind of knowledge would be fundamental to translate into functional product specifications the customers desires and needs and therefore maintain a firm's Innovative Capacity in the market, from a given technological paradigm. Through a case sudy about the development of a best seller popular vehicle in Brazil, this work atempts to discuss how this kind of knowledge could impact on the maintenance of Innovative Capacity in automotive industry. Conclusion suggests that the Architectural Knowledge is a competence that still belongs to Automakers, which allows them to detect customers requirements and translate them into a product. On the other hand, suppliers, in this current technological paradigm would have not this competence.

Key-words: Product Development, Automotive Industry, Architectural Knowledge

\section{Referências Bibliográficas}

BALDWIN,C. e CLARK,K.B. - Managing in the Age of Modularity - Harvard Business Review, V.75, n5, 1987

CLARK, K.B. e FUJIMOTO, T. Product Development Performance - Harvard Business School Press, 1991.

EISENHARDT, K. - Building Theories from Case Study Research - Academy of Management Review. Vol.14, $\mathrm{n}^{\circ} 14, \mathrm{pp}-532-550.1989$ 
FINE,C. Clockspeed - Winning Industry Control in the Age of Temporary Advantage. New York, Perseus Book, 1998.

FINE, C. e WHITNEY, D. - Is the Make or Buy Decision a Core Competence? - IMVP / MIT Working Paper, 1996

FIXSON, S. e SAKO,M. - Modularity in Product Architecture: Will the Auto Industry Follow the Computer Industry? (An Analysis of Product Architecture, Market Conditions and Industrial Forces) - IMVP/ MIT Working Paper, 2001

HENDERSON, R.M. e CLARK, K. - Architectural Innovation: The Reconfiguration of Existing Product technologies and the Failure of Established Firms - Administrative Science Quarterly, 35, 1990

MELlO,A.M.,VASCONCELlOS,L.H.R. e MARX,R. - Estariam as Montadoras Abrindo Mão de Suas Competências Essenciais no Desenvolvimento de Motores? - Um Estudo de Caso do Primeiro Veículo Nacional Bicombustível - XXIX Encontro da ANPAD, Brasília, DF, 2005.

MILES, M. e HUBERMAN, A. Qualitative data analysis: a sourcebook of new methods, Beverly Hills, CA, Sage, 1994.

SAKO, M. - Modulariry and Outsourcing: The nature of Co-evolution of product Architecture and Organisation Architecture in the Global Automotive Industry - Cap. Livro - The Business of Systems Integrations, editado por Prencipe, Davies and Hobday - Oxford University Press, 2002

TAKEISHI, A. - Knowledge Partioning in the Interfirm Division of Labor - Organization Science - Vol.13, n³3, 2002.

ULRICH, K. e ELLISON, D. Beyond Make-buy: Internalization and Integration of Design and Production Working Paper - Dept. of Op.and Inf. Management - The Wharton School, University of Pennsylvania - Jan.2004.

ULRICH, K. e EPPINGER. S - Product Design and Development, Capítulo 7 - McGrawHill, 1995

VOSS, C., TSIKRIKTSIS, N. E FROHLICH, M. - Case Research in Operations Management - International Journal of Operations and Production Management. Vol.22, n², pp-195-219. 2002

YIN, R. Estudo de caso: Planejamento e Métodos, Porto Alegre, Bookman, 2002.

Adriana Marotti de Mello

Escola Politécnica da USP

Departamento de Engenharia de Produção

Doutoranda

Av.Prof.Almeida Prado, trav.2, no.128 - Cidade Universitária - São Paulo - SP CEP 05508-070

Telefones para contato: 30915363 r.423

e-mail:adriana.mello@poli.usp.br

Roberto Marx

Escola Politécnica da USP

Departamento de Engenharia de Produção

Professor Doutor

Av.Prof.Almeida Prado, trav.2, no.128 - Cidade Universitária - São Paulo - SP CEP 05508-070 
Telefones para contato: 30915363 r.423

e-mail:robemarx@usp.br

Recebido para publicação em: 24/04/07

Aceito para publicação em: 21/05/07 